\title{
Three-dimensional simulation of the impregnation stage of the RTM process considering the local structure of the fiber layup
}

\author{
N. Wegh ${ }^{1}$, S. Bachschuster ${ }^{2}$, D. Gaudlitz ${ }^{2} \&$ M. Klein ${ }^{1}$ \\ ${ }^{1}$ Institut für Mathematik und Rechneranwendung LRT-1, \\ Universität der Bundeswehr München, Germany \\ ${ }^{2}$ FluiDyna GmbH, Garching bei München, Germany
}

\begin{abstract}
This paper focuses on the simulation of the impregnation stage of the Resin Transfer Moulding (RTM) process. RTM is a method of producing composite structures by binding the fiber preform with resin within a closed mould. A fiber layup consists of layers of textiles, which may have different material properties. As a result of placing the fiber layup into the mold, the textile layers get deformed and distorted. A key influencing parameter for the resin flow is the textile permeability, which is coupled to the fiber volume ratio (FVR) and is anisotropic in general. Affected by the deformation and distortion, the FVR can vary from nearly zero to a value of approximately 0.6 . Therefore volume-averaged Navier-Stokes equations with variable FVR are developed. The resistance to the flow through the fiber materials, which are modeled as a porous media, is given by Darcy's law. For predicting the macroscopic permeability, a unit-cell impregnation model is introduced. This model takes into account the mesoscopic structure of the porous media, i.e. the roving layout. Since the permeability of a fiber preform is direction-dependent, a transformation with respect to the local fiber orientations of the considered technical component is performed. This implementation allows a three-dimensional simulation of the impregnation stage of the RTM process taking into account the modification of the fiber layup caused by placing it into the mold. The required input will be provided by an external drape simulation, which is however not part of this work. Results will be presented for a eninge bonnet like geometry.
\end{abstract}

Keywords: Resin Transfer Moulding (RTM), permeability, multi-scale modelling, unit-cell, volume averaging method, fabric/textiles. 


\section{Introduction}

Composite structures manufactured by the Resin Transfer Moulding (RTM) process are lightweight structural elements, capable of withstanding high stresses and they are used e.g. in the aerospace or automobile industry. RTM is a method of producing composite structures by binding the fiber preform (typically fiberglass, carbon or aramid) using a resin (epoxies, polyamides, polyesters or phenolics) within a closed mould. To withstand high stresses the quality of the manufacturing of the technical parts is of great importance. The part has to be void free and all fibers need to be wetted by the resin. Therefore knowledge of the resin flow during the injection stage of the RTM process is crucial. Darcy's law is commonly used to describe the flow through a porous media,

$$
q=\frac{-\kappa}{\mu} \nabla P
$$

where $q$ is the so called Darcy flux, $\nabla P$ is the pressure gradient and $\mu$ is the dynamic viscosity of the fluid. The resistance of the porous fiber material to the resin flow is usually expressed in terms of a directional permeability $\kappa$ and is a key influencing parameter. The fluid velocity $u$ is related to the Darcy flux $q$ by

$$
u=\frac{q}{\phi},
$$

where $\phi$ is the porosity, which can be defined as $\phi=\frac{V}{V_{t}}$. In this equation $V_{t}$ is the total volume and $V$ is the volume of the voids. A fiber layup consists of layers of textiles, which may have different material properties. The textile permeability is in general anisotropic and coupled to the fiber volume ratio $(=1-\phi)$. When placing the fiber layup into the mold, the textile layers get deformed resulting in local fiber volume ratio (FVR), which may vary from nearly zero (e.g. at corners) to approximately 0.6 . In a microstructural framework, where each filament of a roving is resolved, the resin flow is described by the Navier-Stokes equations. For a three-dimensional simulation of the impregnation stage of the RTM process Navier-Stokes equations including a macroscopic description of fiber materials by porous media of corresponding properties are needed. Individual rovings or even filaments can not be considered in this context. The macroscopic fluid velocity $u$ is coupled to the FVR (Eq. (2)) since only a fraction of the total volume is available for fluid flow. The resistance to the flow caused by fiber materials, the sink term in the macroscopic Navier-Stokes equations, is modeled with the Darcy equation (Eq. (1)). For Darcy's law the permeability $\kappa$ is required.

The permeability is a local parameter of the textile and depends e.g. on the geometry and the orientation of the fibers. One can obtain the permeability by experimental investigations. To fully determine the permeability tensor for one 
mechanical condition of the fiber material (grade of shearing and compression, etc.), which might be obtained by a draping process, three injection experiments have to be carried out with the direction of injection (e.g. the pressure gradient for injection) aligned with one of the spatial directions, respectively. Hence, a large amount of experimental work has to be done in order to characterize the prevailing permeabilities of a fiber material for an application. An alternative is to determine the permeability by simulations. To fully take into account the microstructure of the textile, a very fine computational mesh would be needed, leading to an unacceptable amount of computational costs. Since we are interested in the macroscopic resin flow the determined permeability should take into account the mesostructure of the fiber material, i.e. the orientation, the cross-sectional shape and the way of weaving of the rovings. Therefore a known expression for calculating the permeability of uni-directional (UD) fibers is used inside the rovings, which are composed by aligned filaments (microstructure). Due to the regularity of the textile, the permeability is predicted by employing an unit-cell impregnation model. Using known local parameters like shear angle, compression and orientation of the rovings, the permeability of the fiber material can be provided as an input parameter for the macroscopic sink term (Darcy's law).

The paper is structured as follows. In Section 2 the multiphase flow model is presented. The different unit-cell models and the resulting permeability tensor are explained in Section 3. Section 4 exhibits some simulation results with our software. Finally, some remaining issues are discussed and the conclusion is given.

\section{Multiphase flow model}

We consider a two phase fluid flow in a porous media. In particular this means that we have three phases $k: f$ fluid, $g$ gas and the solid matrix $s$. At the microstructure level the flow of phase $k$ is described by the following mass and momentum equations:

$$
\begin{array}{cl}
\text { Mass } & \frac{\partial \rho_{k}}{\partial t}+\nabla \cdot\left(\rho_{k} \vec{v}_{k}\right)=0 \\
\text { Momentum } & \frac{\partial}{\partial t}\left(\rho_{k} \vec{v}_{k}\right)+\nabla \cdot\left(\rho_{k} \vec{v}_{k} \vec{v}_{k}\right)=-\nabla P+\nabla \cdot \tau_{k}+\vec{F}_{k}
\end{array}
$$

where $\tau=\mu_{k}\left(\nabla \vec{v}_{k}+\left(\nabla \vec{v}_{k}\right)^{T}\right)$ is the shear stress tensor with viscosity $\mu_{k}, \vec{F}$ is the body force and $\vec{v}_{k}$ is the velocity vector of phase $k$ at micro scale. Since knowledge of the resin flow at macro scale is needed, to avoid voids and to guarantee that the resin is well distributed, the set of equations (3) and (4) is homogenized. We provide a brief review of the idea of volume-averaging techniques by following Whitaker [1] and Bear [2]. For more details the reader is referred to the references [3-5]. We take a representative elementary volume occupied by the three phases, whose interfaces may move with time

$$
V_{0}=V_{f}(t)+V_{g}(t)+V_{s}(t) .
$$


The volume $V_{0}$ is defined as an arbitrary volume chosen large enough to be statistically representative and small enough to regard spatial variance in the porosity. The definition of the superficial volume average $\langle\theta\rangle_{0}$ and the intrinsic volume average $\langle\theta\rangle_{k}$ of a transport quantity $\theta$ in phase $k$ is given as

$$
\langle\theta\rangle_{0}=\frac{1}{V_{0}} \int_{V_{0}} \theta \xi_{k} d V, \quad\langle\theta\rangle_{k}=\frac{1}{V_{k}} \int_{V_{0}} \theta \xi_{k} d V
$$

here $\xi_{k}$ denotes the phase function and $V_{k}$ is the volume fraction of the total volume $V_{0}$ occupied by phase $k$. The phase function $\xi_{k}$ has the value 1 in the phase $k$ and zero elsewhere. The deviation of the intrinsic volume average is defined as $\{\theta\}=\theta-\langle\theta\rangle_{k}$. It is zero when $\theta$ is uniformly distributed. An important relationship is the following $\langle\theta\rangle_{0}=\epsilon_{k}\langle\theta\rangle_{k}$, where $\epsilon_{k}=V_{k} / V_{0}$ is the part of the volume filled with phase $k$. The porosity $\phi=\epsilon_{f}+\epsilon_{g}$ is assumed to be constant with respect to time $\left(\frac{\partial \phi}{\partial t}=0\right)$ but not spatially constant. Applying the averaging formulae, Eqs (3) and (4) obtain the following form:

$$
\begin{gathered}
\text { Mass }\left\langle\frac{\partial \rho_{k}}{\partial t}\right\rangle_{0}+\left\langle\nabla \cdot\left(\rho_{k} \vec{v}_{k}\right)\right\rangle_{0}=\langle 0\rangle_{0} \\
\text { Momentum }\left\langle\frac{\partial}{\partial t}\left(\rho_{k} \vec{v}_{k}\right)\right\rangle_{0}+\left\langle\nabla \cdot\left(\rho_{k} \vec{v}_{k} \vec{v}_{k}\right)\right\rangle_{0}=\langle-\nabla P\rangle_{0}+\langle\nabla \cdot \tau\rangle_{0}+\langle\vec{F}\rangle_{0} .
\end{gathered}
$$

The following assumptions are made in this paper:

- The control volume is stationary. Since $S_{f f}$ and $S_{g g}$, the interfaces on the respective surface (here fluid-fluid or gas-gas) coincide with the outer boundary of the control volume, $\vec{\omega}_{f f}$ or $\vec{\omega}_{g g}$, the velocity of microscopic points on the respective surface is equal to zero.

- The fluid and the gas are incompressible. The density is assumed to be constant with respect to time and space and thus $\left\{\rho_{k}\right\}$ is zero.

- The porous structure is rigid (constant with respect to time) and has the noslip boundary condition. This means $\vec{\omega}_{f s}$ and $\vec{\omega}_{g s}$ are equal to the velocity $\vec{v}_{f}$ and $\vec{v}_{g}$ at the fluid/gas-solid interface. Additionally, the porous structure is stationary, therefore $\vec{\omega}_{f s}=\vec{\omega}_{g s}=0$.

- The fluid and gas phase are Newtonian fluids, this means $\mu_{f}$ and $\mu_{g}$ are constant and the deviation $\left\{\mu_{k}\right\}$ is zero.

With these assumptions the averaging rules, as derived by Slattery [6], Whitaker [1] and Bear [2], are applied to Eqs (5) and (6). Then for the fluid phase the equations read

$$
\text { Mass } \nabla \cdot\left(\epsilon_{f}\left\langle\vec{v}_{f}\right\rangle_{f}\right)=0
$$




$$
\begin{aligned}
\text { Momentum } & \frac{\partial}{\partial t}\left(\epsilon_{f}\left\langle\rho_{f}\right\rangle_{f}\left\langle\vec{v}_{f}\right\rangle_{f}\right)+\nabla \cdot\left(\epsilon_{f}\left\langle\rho_{f}\right\rangle_{f}\left\langle\vec{v}_{f}\right\rangle_{f}\left\langle\vec{v}_{f}\right\rangle_{f}\right) \\
& +\nabla \cdot\left(\epsilon_{f}\left\langle\left\langle\rho_{f}\right\rangle_{f}\left\{\vec{v}_{f}\right\}\left\{\vec{v}_{f}\right\}\right\rangle_{f}\right)+\frac{1}{V_{0}} \iint_{S_{f g}}\left(\rho_{f} \vec{v}_{f}\right)\left(\vec{v}_{f}-\vec{\omega}_{f g}\right) \cdot n d S \\
& =\epsilon\langle P\rangle_{f}+\frac{1}{V_{0}} \int_{S_{f s}}\{P\} n d S+\frac{1}{V_{0}} \iint_{S_{f g}}\{P\} n d S \\
& +\nabla \cdot\left(\epsilon_{f}\langle\mu\rangle_{f}\left(\nabla\left\langle\vec{v}_{f}\right\rangle_{f}+\frac{1}{V_{f}} \iint_{S_{f g}} \vec{v}_{f} n d S\right)\right) \\
& +\frac{1}{V_{0}} \int_{S_{f s}}\left(\mu \nabla \vec{v}_{f}\right) \cdot n d S+\frac{1}{V_{0}} \iint_{S_{f g}}\left(\mu \nabla \vec{v}_{f}\right) \cdot n d S+\langle\vec{F}\rangle_{0} .
\end{aligned}
$$

The Eqs (7), (8) and all subsequent equations look the same for the gas phase with the corresponding indices. Following Bear and Bachmat [4], it may be assumed that $\left|\left\langle\left\langle\rho_{f}\right\rangle_{f}\left\{\vec{v}_{f}\right\}\left\{\vec{v}_{f}\right\}\right\rangle_{f}\right| \ll\left|\left\langle\left\langle\rho_{f}\right\rangle_{f}\left\langle\vec{v}_{f}\right\rangle\left\langle\vec{v}_{f}\right\rangle\right\rangle_{f}\right|$ and therefore the corresponding term in Eq. (8) will be disregarded.

For passing over to the one fluid formulation, Eqs (7) and (8) for the fluid phase and the gas phase are summed up. Let us consider the surface integrals (momentum Eq. (8)). There are two kinds of boundaries (fluid-gas surfaces and fluid/gassolid surfaces). The interaction between fluid/gas with the solid, represented by the surface integrals $S_{k s}$ for $k=f, g$, together with the body force $\vec{F}$ can be interpreted, following [3], as Darcy's law. In the governing Eqs (10) Darcy's law is written as the sink term $S=-\left(\frac{\mu}{\kappa}\right) \vec{u}$, since the equation implies a pressure drop. The surface integrals $S_{f g}$ in Eq. (8) of the fluid-gas surface combined represent the surface tension [7,8]. However, in this paper the surface tension is not considered. Defining a mixture density $\rho$ and a center-of-mass velocity $\vec{u}$

$$
\rho=\epsilon_{f} \rho_{f}+\epsilon_{g} \rho_{g}, \quad \vec{u}=\frac{1}{\rho}\left(\epsilon_{f} \rho_{f} \vec{v}_{f}+\epsilon_{g} \rho_{g} \vec{v}_{g}\right)
$$

and introducing these definitions into the one fluid formulation reduces it to the compact form

$$
\begin{aligned}
& \operatorname{Mass} \nabla \cdot(\phi \vec{u})=0 \\
& \begin{aligned}
\text { Momentum } & \frac{\partial}{\partial t}(\phi \rho \vec{u})+\nabla \cdot(\phi \rho \vec{u} \vec{u})=\phi \nabla P+\nabla \cdot(\phi \mu \nabla \vec{u}) \\
& +\nabla(\phi \vec{u}) \cdot \nabla(\mu)-\left(\frac{\mu}{\kappa}\right) \vec{u} .
\end{aligned}
\end{aligned}
$$

To exclude misunderstandings all variables are homogenized by the volume averaging method with spatially variable porosity $\phi$. These governing equations (Eqs (9) and (10)) are implemented in OpenFOAM [9]. A closure for the jump 
terms occurring in the one fluid formulation is not known in the literature. Therefore these terms are neglected according to $[3,7,8]$.

\section{Modeling permeability}

The aim of the macroscopic flow simulation of the impregnation process is to ensure the quality of the composite material. In the previous section we introduced the volume-averaged Navier-Stokes equations. As an input parameter a local (macroscopic) permeability for every grid cell is needed. Simulations of resin flow through characteristic unit-cells of specific structure are carried out, see e.g. Fig. 2(a), to derive properties of corresponding porous media. The required geometrical parameters for the unit-cell model like the roving orientation, shear angle, thickness of the layer and the FVR are assumed to be provided by a draping simulation, which is not part of this work. Following Darcy's law (Eq. (1))

$$
\left(\begin{array}{c}
q_{x} \\
q_{y} \\
q_{z}
\end{array}\right)=\frac{1}{\mu}\left(\begin{array}{l}
\kappa_{x x} \frac{\partial P}{\partial x}+\kappa_{x y} \frac{\partial P}{\partial y}+\kappa_{x z} \frac{\partial P}{\partial z} \\
\kappa_{y x} \frac{\partial P}{\partial x}+\kappa_{y y} \frac{\partial P}{\partial y}+\kappa_{y z} \frac{\partial P}{\partial z} \\
\kappa_{z x} \frac{\partial P}{\partial x}+\kappa_{z y} \frac{\partial P}{\partial y}+\kappa_{z z} \frac{\partial P}{\partial z}
\end{array}\right),
$$

here in tensor notation, all components of the permeability tensor $\kappa$ for a saturated porous medium can be determined by performing three stationary flow simulations. By applying a prescribed mass flow $q$ in one spatial direction and periodic boundary conditions for the two remaining spatial directions, the pressure loss, or pressure gradient, in the main flow direction can be obtained. By performing two additional flow simulations of similar setup, where only the direction of the main flow has been changed, all components $\kappa_{i j}$ of the permeability tensor can be determined. In doing so, the main diagonal contains the important information and the off-diagonal terms should be nearly zero.

In order to limit the computational costs, the flow inside the rovings (microstructure), which are composed of aligned filaments, is modeled by a porous media consisting of unidirectional fibers. The permeability of this UD-structure can be determined employing the model of Gebart [10], which takes into account either quadratic or hexagonal fiber packing, geometric dimensions of the filaments in the roving and the porosity. The permeabilities are predicted as follows

$$
\kappa_{\mid}=\frac{8 R^{2}}{c} \frac{\left(1-V_{f}\right)^{3}}{V_{f}^{2}}, \quad \kappa_{\perp}=C_{1}\left(\sqrt{\frac{V_{f_{\max }}}{V_{f}}-1}\right)^{5 / 2} R^{2},
$$

where $\kappa_{\mid}$describes the permeability along the fibers and $\kappa_{\perp}$ describes the permeability perpendicular to the fibers. In this equations $R$ is the radius of the filaments, $V_{f}$ is the fiber volume with its theoretical maximum $V_{f_{\max }}$, and $c$ is a shape factor. The parameters $C_{1}$ and $V_{f_{\max }}$ depend on the fiber packing (quadratic or hexagonal). The model parameters are summarized in Table 1.

In this paper the biaxial carbon fabric HPT-610 (SGL Carbon) having roving angles of $\pm 45^{\circ}$ is examined. The material data is summarized in Table 2. A 
Table 1: Parameter for the permeability model of UD-fabrics according to Gebart.

\begin{tabular}{c|ccc}
\hline Fiber packing & $C_{1}$ & $V_{f_{\max }}$ & $c$ \\
\hline Quadratic & $\frac{16}{9 \pi \sqrt{(2)}}$ & $\frac{\pi}{4}$ & 57 \\
Hexagonal & $\frac{16}{9 \pi \sqrt{(6)}}$ & $\frac{\pi}{2 \sqrt{(3)}}$ & 53 \\
\hline
\end{tabular}

Table 2: Material data of the fabric HPT-610.

\begin{tabular}{c|cc}
\hline & $\phi$ & $R$ \\
\hline Roving & 0.207 & $710^{-6} \mathrm{~m}$ \\
Sewing thread & 0.5 & $10^{-5} \mathrm{~m}$ \\
\hline
\end{tabular}

computed tomography (CT) scan of HPT-610 (Fig. 1) shows two layers of rovings aligned perpendicular to each other of half elliptical shape and a sewing thread is clearly visible. For generating a unit-cell, the CT-scan delivers a far too complex geometry, featuring details like individual filaments. Based on this data, the generation of approximate computer meshes to be used for finite-volumebased CFD-tools is very challenging. Therefore a simpler approach is sought. The simplest unit-cell model is the "brick-model" (Fig. 2(a)) with rovings of rectangular cross section and without sewing thread. In all figures of unit-cell models presented in this paper, the red area denotes the $+45^{\circ}$ oriented fiber and the green area the $-45^{\circ}$ oriented fiber. The sewing thread is shown in blue and the channel between the rovings is highlighted in yellow. The roving permeability is computed by Eqs (3) by using the material parameters given in Table 2 and assuming a hexagonal fiber packing.

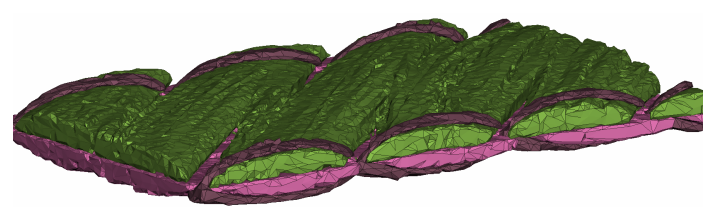

Figure 1: CT-Scan of HPT-610 fabric specimen by H. Finckh, ITV Denkendorf.

We start with the examination of the influence of the sewing thread on the macroscopic permeability. Since considerable flow is expected to run through the channels between the rovings and the sewing threads are present blocking some of them, the permeability should decrease. The question is, how much influence has the sewing thread on the permeability and is it negligible. In contrast to Fig. 2(a), 
the second "brick-model" (Fig. 2(b)) contains a sewing thread. The sewing thread is considered also as an UD reinforcement and therefore its permeability is predicted by Gebarts model. Again we presume a hexagonal packing and use the parameter from Table 2. To investigate the influence of deformation and distortion, which are a result of the draping, the unit-cell was sheared. By shearing the setup of the rovings, the channels between the rovings obtain a different shape. Due to this it should have a great influence on the textile permeability in the horizontal. The effect on the permeability in the perpendicular ( $y$-axis), since there is no major geometrical change, should be small. For the first analysis of the effect of distortion, the rovings are arranged at $\mathrm{a} \pm 30^{\circ}$ angle to the middle $\left(60^{\circ}\right.$ angle to each other). A better approximation of the CT-Scan of HPT-610 (Fig. 1) is the ellipse-model (Fig. 4) with two layers of rovings right-angled to each other of half elliptical shape and with a sewing thread. Due to the elliptical shape of the rovings, the separating channel between them is larger, the mass flow in this channel will increase and the permeability rises (see Table 3).

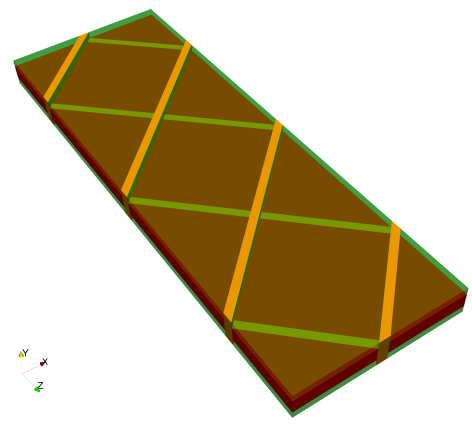

(a) without sewing thread

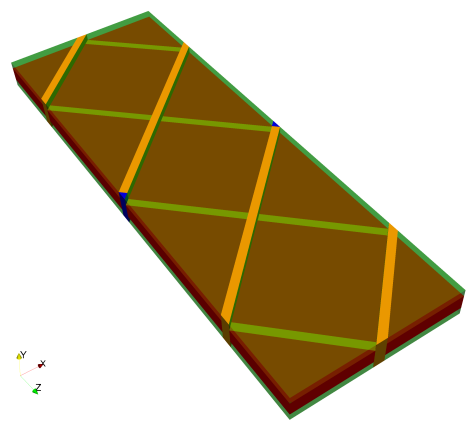

(b) with sewing thread

Figure 2: "Brick-model" of a unit-cell of the fabric HPT-610. (Red: fiber $+45^{\circ}$; green: fiber $-45^{\circ}$; blue: sewing thread; yellow: channel between the rovings.)

Table 3 compares the calculated permeabilities for all presented models in this work. The suffix of the permeability tensor in this table denotes the angle between rovings and center, e.g. \pm 45 is the undistorted HPT-610. As assumed, the offdiagonal entries are small compared to the main diagonal and therefore the main entries are highlighted.

It starts with the tensor for the brick-model with (Fig. 2(b)) and without (Fig. 2(a)) sewing thread and it can be clearly seen that the sewing thread has an impact on the permeability tensor. The $\kappa_{y y}$ entry without sewing thread is by 10 percent larger then with the sewing thread. This is considered a non negligible influence so that it should always be considered.

The results (see Table 3 among $\kappa_{ \pm 30}$ ) of the sheared brick-model (Fig. 3) confirm our expectation. The permeability tensor entry $\kappa_{y y}$ is unaffected in contrast to the entries $\kappa_{x x}$ and $\kappa_{z z}$. The horizontal entries differ by $-44 \%$ in 


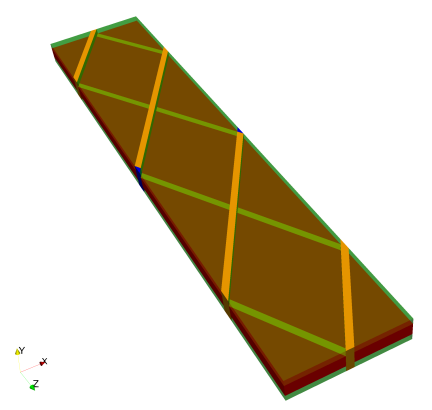

Figure 3: Brick-model distorted fiber angel $\pm 30^{\circ}$. Red: fiber $+45^{\circ}$; green: fiber $-45^{\circ}$; blue: sewing thread; yellow: channel between the rovings.

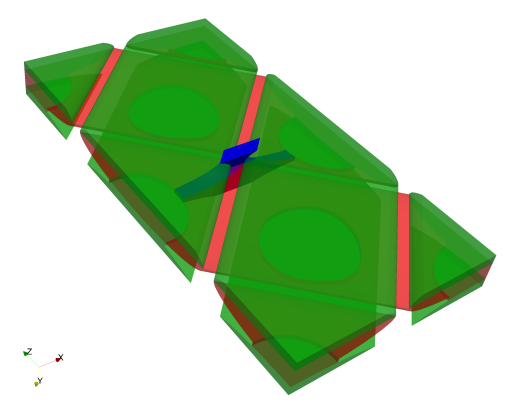

Figure 4: Ellipse-model fiber angel $\pm 45^{\circ}$. Red: fiber $+45^{\circ}$; green: fiber $-45^{\circ}$; blue: sewing thread; yellow: channel between the rovings.

Table 3: Comparison of the permeability of the different models.

$$
\begin{aligned}
& \kappa_{ \pm 45} \text { with sewing thread } \\
& \left(\begin{array}{ccc}
\mathbf{3}, \mathbf{4} \cdot \mathbf{1 0}^{-\mathbf{1 1}} & 2,8 \cdot 10^{-14} & 3,0 \cdot 10^{-14} \\
7,1 \cdot 10^{-16} & \mathbf{5 , 7} \cdot \mathbf{1 0}^{-\mathbf{1 2}} & 2,3 \cdot 10^{-16} \\
7,7 \cdot 10^{-14} & 3,3 \cdot 10^{-14} & \mathbf{3}, \mathbf{3} \cdot \mathbf{1 0}^{-\mathbf{1 1}}
\end{array}\right) \\
& \kappa_{ \pm 45} \text { without sewing thread } \\
& \left(\begin{array}{ccc}
\mathbf{3}, \mathbf{5} \cdot \mathbf{1 0}^{-\mathbf{1 1}} & 4,7 \cdot 10^{-14} & 2,5 \cdot 10^{-14} \\
1,1 \cdot 10^{-15} & \mathbf{6 , 3} \cdot \mathbf{1 0} \mathbf{1 0}^{-\mathbf{1 2}} & 3,5 \cdot 10^{-16} \\
8,2 \cdot 10^{-16} & 1,8 \cdot 10^{-13} & \mathbf{3}, \mathbf{5} \cdot \mathbf{1 0}^{-\mathbf{1 1}}
\end{array}\right) \\
& \kappa_{ \pm 30} \text { with sewing thread } \\
& \left(\begin{array}{ccc}
\mathbf{1}, \mathbf{8 6} \cdot \mathbf{1 0}^{-\mathbf{1 1}} & 1,3 \cdot 10^{-14} & 9,2 \cdot 10^{-13} \\
4,1 \cdot 10^{-16} & \mathbf{5 , 7} \cdot \mathbf{1 0}^{-\mathbf{1 2}} & 1,6 \cdot 10^{-16} \\
3,6 \cdot 10^{-13} & 2,1 \cdot 10^{-14} & \mathbf{4 , 8} \cdot \mathbf{1 0} \mathbf{0}^{-\mathbf{1 1}}
\end{array}\right) \\
& \kappa_{ \pm 45} \text { Ellipse-model } \\
& \left(\begin{array}{ccc}
\mathbf{3}, \mathbf{5} \cdot \mathbf{1 0}^{-\mathbf{1 0}} & 2,3 \cdot 10^{-14} & 1,8 \cdot 10^{-15} \\
3,4 \cdot 10^{-14} & \mathbf{1 , 0} \cdot \mathbf{1 0}^{-\mathbf{1 1}} & 1,6 \cdot 10^{-15} \\
2,3 \cdot 10^{-15} & 7,1 \cdot 10^{-15} & \mathbf{2 , 7} \mathbf{7} \mathbf{1 0}^{-\mathbf{1 0}}
\end{array}\right)
\end{aligned}
$$

$x$-direction and by $+45 \%$ in $z$-direction, this means the associated macroscopic flow is increased in $z$-direction and is reduced in $x$-direction. In order to ensure a realistic resin flow simulation, the great influence of deformation and distortion on the textile permeability should be considered. 
The last permeability tensor belongs to the ellipse-model (Fig. 4). The influence is about one order of magnitude in $x-/ z$-direction and about a half an order of magnitude in $y$-direction.

In order to keep the runtime of a simulation of the impregnation stage of a composite structure small, the permeability tensors are stored at a database. This means the simulation of a permeability tensor has to be done only once for each considered textile for different shears and compressions.

Finally, after determining the permeability a coordinate transformation of the calculated tensor along the fiber orientation into the global coordinate system is carried out in OpenFOAM.

\section{Validation and application}

We start with a verification of the implemented governing equations (9), (10). The sink term in (10) is disregarded. In a first step a flow through a pipe was simulated with constant inlet volume flow $\left(0.1 \frac{\mathrm{m}}{\mathrm{s}}\right)$ and zero gradient boundary conditions for the velocity on the cylinder wall. The pipe (Fig. 5(a)) is split up into four segments with different porosity and length. The first and last section have the same length $(l=0.1 \mathrm{~m})$ and same porosity $(\phi=1)$. The Second and the third area are both $0.4 \mathrm{~m}$ long but in section number two only one half of the total volume is available for fluid flow $(\phi=0.5)$ and in the third only one-fourth $(\phi=0.25)$. Analytically, the flow front should need $1 \mathrm{~s}$ for the first, third and last section to traverse, since the velocity $u=\frac{q}{\phi}$ equals $0.1 \frac{\mathrm{m}}{\mathrm{s}}$ in the first and last segment and equals $0.4 \frac{\mathrm{m}}{\mathrm{s}}$ in the third. The velocity in the second area is twice the inflow speed $\left(0.2 \frac{\mathrm{m}}{\mathrm{s}}\right)$ consequently it should need $2 \mathrm{~s}$. In total the resin is supposed to passe through the cylinder in $5 \mathrm{~s}$. In the diagram (Fig. 5(b)) the velocity of the simulation and the analytical solution are shown. The simulated velocity matches very well with the analytical solution as well as the predicted passing time for the total cylinder and for each segment. In the second verification step the implementation of Darcy's law was verified by comparing predicted and theoretical pressure drop in a pipe. (Results are not shown here.)

Next we show a feasibility study of an injection simulation including a detailed fiber layup. The geometry is a flat plate of dimensions $1.5 \mathrm{~m} \times 1.5 \mathrm{~m} \times 2.1 \mathrm{~mm}$ (Fig. 6(a)), which is considered to be a simple representation of an engine bonnet. The fiber layup consists of three UD-layers of HPT- 610 rotated by $60^{\circ}$. Each layup is represented by three cells in height, so the whole composite structure is nine cells high. As inlet a central injection port $(2 \mathrm{~cm} \times 2 \mathrm{~cm})$ was used and outflow was realised at the edges. Horizontal cuts in all cells (Fig. 6(b)) show a realistic flow front at $t=27 \mathrm{~s}$. The different preferred flow directions in each fiber layer caused by the rotation of the permeability tensor are clearly visible. A more realistic setup for the engine bonnet is depicted in Fig. 7(a). The orientation of the fiber layup follows the geometry of the curved geometry. It consists of three UD-layers of HPT-610 in height. In contrast to the previous case only the middle layers are rotated by $45^{\circ}$ in plane. The preferred flow direction in the rotated layer is pointing 

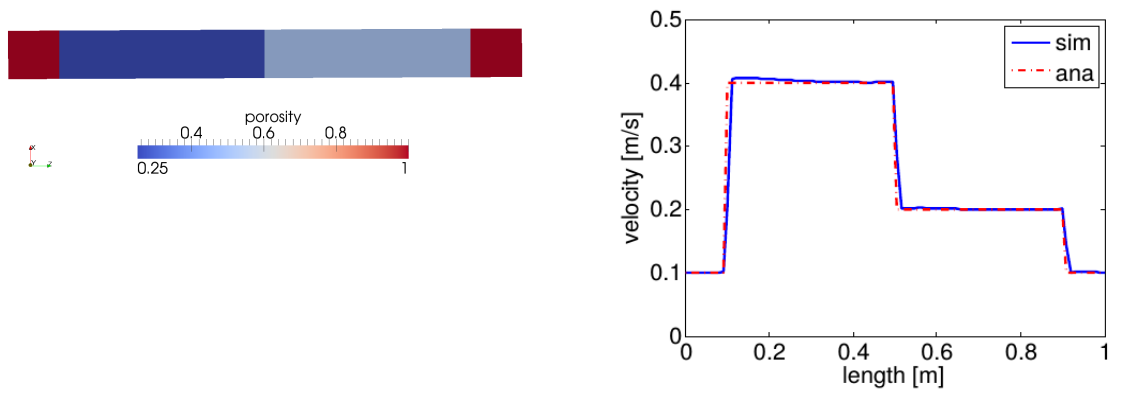

(a)

(b)

Figure 5: (a) Pipe with four porosity segments. (b) Diagram of the simulated velocity and analytical solution.

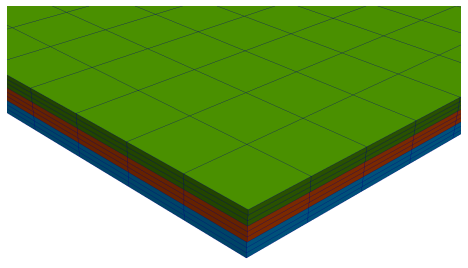

(a)

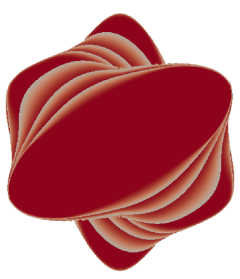

(b)

Figure 6: (a) Fiber layup with 3 UD-layers rotated by $60^{\circ}$. (b) Horizontal cuts in all cells showing the flow front at $t=27 \mathrm{~s}$.

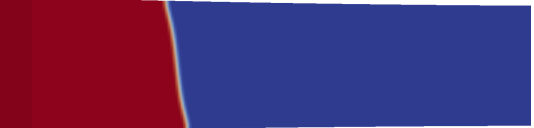

(b)

Figure 7: (a) Geometry of the curved engine bonnet. (b) Flow front at $t=14 \mathrm{~s}$ from underneath (laterally reversed).

to the back side. Due to the rotation of the middle fiber layer, the flow front on the back side is ahead of the one on the front side (Fig. 7(a), Fig. 8). 


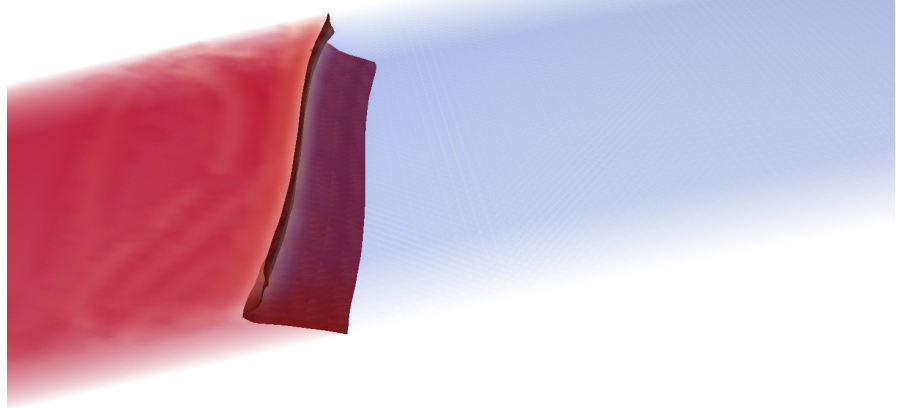

Figure 8: Flow front of Fig. 7 at $t=14 \mathrm{~s}$.

\section{Remaining issues and conclusion}

\subsection{Remaining issues}

For better estimation of the influence of shear and compression on the permeability, we are working on several unit-cell setups with different textiles in order to setup a database. Considering the effects of dual-scale flow process (unsaturated/saturated tows), for more details see [11,12], is also work in progress. For validation of the unit-cell model the simulated permeability will be compared to experimental data of an undistorted textile. Finally comparison of experimental results for a realistic demonstrator with our implementation coupled with a drape simulation is planned.

\subsection{Conclusion}

We have implemented macroscopic Navier-Stokes equations with spatial variable porosity. The meso scale permeability of textile reinforcements is an important input parameter for these equations. Due to the lack of experimental data, we introduced an unit-cell impregnation model. Based on a CT-scan of the HPT610 fabric specimen, we generated different unit-cell settings and demonstrated the influence of a sewing thread and distortion on the fiber layup. In order to keep the computational cost of the simulation moderate, we propose to use a database, that contains the permeability tensor for different textiles as a function of local compression and shear. To fully take the local structure of the textile into account, a coordinate transformation of the permeability along the fiber orientation is carried out. It was shown that our approach (Navier-Stokes equations + unitcell permeability) allows a three-dimensional simulation of the impregnation stage of the RTM process with respect to the modification of the fiber layup caused by placing it into the mold. Therefore this implementation can be coupled with an external drape simulation. 


\section{Acknowledgement}

The authors thank Hermann Finckh from the Institut für Textil- und Verfahrenstechnik (ITV) Denkendorf for providing the CT-Scan (Fig. 1).

\section{References}

[1] Whitaker, S., The method of volume averaging, volume 13. Springer, 1998.

[2] Bear, J., Dynamics of fluids in porous media. Elsevier, New York, 764p, 1972.

[3] Nield, D.A. \& Bejan, A., Convection in porous media. Springer, 2006.

[4] Bear, J. \& Bachmat, Y., Introduction to modeling of transport phenomena in porous media, volume 4. Springer, 1990.

[5] Gray, W. \& Lee, P., On the theorems for local volume averaging of multiphase systems. International Journal of Multiphase Flow, 3(4), pp. 333-340, 1977.

[6] Slattery, J.C., Single-phase flow through porous media. AIChE Journal, 15(6), pp. 866-872, 1969.

[7] Sabisch, W., Wörner, M., Grötzbach, G. \& Cacuci, D.G., Dreidimensionale numerische simulation von aufsteigenden einzelblasen und blasenschwärmen mit einer volume-of-fluid-methode. Chemie Ingenieur Technik, 73(4), pp. 368-373, 2001.

[8] Kleinstreuer, C., Two-phase flow: theory and applications. CRC Press, 2003.

[9] Weller, H.G., Tabor, G., Jasak, H. \& Fureby, C., A tensorial approach to computational continuum mechanics using object-oriented techniques. Computers in Physics, 12(6), pp. 620-631, 1998.

[10] Gebart, B., Permeability of unidirectional reinforcements for RTM. Journal of Composite Materials, 26(8), pp. 1100-1133, 1992.

[11] De Parseval, Y., Pillai, K. \& Advani, S., A simple model for the variation of permeability due to partial saturation in dual scale porous media. Transport in Porous Media, 27(3), pp. 243-264, 1997.

[12] Kuentzer, N., Simacek, P., Advani, S.G. \& Walsh, S., Permeability characterization of dual scale fibrous porous media. Composites Part A: Applied Science and Manufacturing, 37(11), pp. 2057-2068, 2006. 